\section{Severe Case of Rickettsiosis Identified by Metagenomic Sequencing, China}

\author{
Zhongqiu Teng, ${ }^{1}$ Yan Shi, ${ }^{1}$ Yao Peng, ${ }^{1}$ Huayi Zhang, \\ Xia Luo, Xinchang Lun, Lianxu Xia, Yuanhai You, \\ Zhenpeng Li, Wen Zhang, Ying Zhang, Shicun Dong, \\ Wentao Guo, Biao Kan, Bo Pang, Jianguo Xu, \\ Aiping Qin
}

\begin{abstract}
Author affiliations: National Institute for Communicable Disease Control and Prevention, Chinese Center for Disease Control and Prevention, Beijing, China (Z. Teng, Y. Peng, X. Luo, X. Lun, L. Xia, Y. You, Z. Li, W. Zhang, B. Kan, B. Pang, J. Xu, A. Qin); Qinghai Center for Disease Control and Prevention, Xining, China (Y. Shi, H. Zhang, S. Dong); Baotou Medical College, Baotou, China (Y. Zhang); Institute of Endemic Diseases of Qinghai, Xining (W. Guo)
\end{abstract}

\section{DOI: https://doi.org/10.3201/eid2705.203265}

A case of Rickettsia sibirica subspecies sibirica BJ-90 infection in China was identified by metagenomic analysis of an eschar biopsy specimen and confirmed by nested PCR. Seroprevalence of spotted fever group Rickettsia was $\approx 17.4 \%$ among the local population. This report highlights the threat of rickettsioses to public health in the Qinghai-Tibet Plateau.

$R$ ickettsia, mainly transmitted by ticks, are a group of obligate gram-negative bacteria that cause mild to life-threatening rickettsioses. Two main groups of Rickettsia have been described on the basis of genetic differences and pathology, spotted fever group (SFG) and typhus group (TG). In China, 5 members of SFG have been identified in human cases (1-4), and 7 kinds of Rickettsia have been detected from ticks or animals in the Qinghai-Tibet Plateau, including R. heilongjiangensis, $R$. raoultii, $R$. slovaca, and $R$. sibirica, which are known to be pathogenic to humans (5-7). However, clinical cases have not been reported. Thus, rickettsioses are probably neglected by local physicians and public health officers. We report a severe case of R. sibirica subspecies sibirica BJ-90 infection in this region.

A 50-year-old herdsman from Zhamashi, Qinghai Province, China, was hospitalized on July 13, 2018, because of intensive intermittent headache, anorexia, and chest tightness. On his fifth day of sheep shearing (designated as day 1), a blood-fed tick had been found on his head. The tick was removed by hand but its mouth parts remained in the man's scalp. The next

${ }^{1}$ These authors contributed equally to this article. day, he became ill with fever, myalgia, itchiness, and asthenia. On day 5, his symptoms intensified and included severe intermittent headaches, which lasted for $\approx 10$ minutes at each onset; high fever, up to $39.5^{\circ} \mathrm{C}$; and fatigue, palpitation, nausea, and vomiting. Erythematous rashes appeared on his trunk, all 4 limbs, and the area behind the ears. Because signs of neurologic dysfunction, including confusion, drowsiness, and delirium appeared, he sought care at Qilian County Hospital on day 9, where he was treated for infectious endocarditis for 3 days before transfer to Qinghai State Hospital. During his visit at the Qinghai State Hospital, he was conscious and alert. Erythematous macules were observed over his trunk, elbow, and lower limbs. A $1.5 \times 1.1 \mathrm{~cm}^{2}$ black eschar was visible at his right posterior occipital bone area; no tenderness was reported (Appendix Figure 1, https:/ / wwwnc.cdc.gov/ EID/article/27/5/20-3265-App1.pdf). The eschar was surgically excised on day 16. No lymphadenopathy was found.

Alterations of the patient's blood biochemistry included increased neutrophils $(88.5 \%$ [reference $45 \%-75 \%$ ]); decreased lymphocytes (9.3\% [reference $20 \%-50 \%$ ]), eosinophils ( $0 \%$ [reference $0.4 \%-8 \%$ ]), and monocytes $(1.9 \%$ [reference $3 \%-10 \%$ ]); elevated creatine kinase-MB (42 U/L [reference 0-25 U/L]) and lactate dehydrogenase (445 U/L [reference 110$245 \mathrm{U} / \mathrm{L}])$; and highly increased C-reactive protein (97.1 mg/dL [reference $0-5 \mathrm{mg} / \mathrm{dL}$ ]), procalcitonin (0.433 ng/dL [reference 0-0.046 ng/dL]), D-dimers $(12.28 \mu \mathrm{g} / \mathrm{mL}$ [reference $0-1.5 \mu \mathrm{g} / \mathrm{mL}$ ]), fibrinogen degradation products $(25 \mu \mathrm{g} / \mathrm{mL}$ [reference $0-5 \mu \mathrm{g} /$ $\mathrm{mL}$ ]), and $\beta$-microglobulin $(4.5 \mu \mathrm{g} / \mathrm{mL}$ [reference $0.8-$ $1.8 \mu \mathrm{g} / \mathrm{mL}])$. The patient was prescribed levofloxacin lactate $(0.5 \mathrm{~g} / \mathrm{d}$ for $6 \mathrm{~d})$. His symptoms subsided, and he was discharged on day 20.

On the basis of tick-bite history and the triad clinical characteristics (fever, rash, and eschar), nested PCR targeting the rickettsial citrate synthase conserved gene (gltA) was performed by using the eschar DNA as a template (Appendix). The 547-bp amplicon sequence shared $100 \%$ identity to $R$. sibirica 246, R. sibirica subsp. sibirica BJ-90, and R. sibirica subsp. mongolitimonae HA-91. The eschar DNA was sequenced by next-generation sequencing (BGI Genomics, https://www.bgi.com). A total of $21.6 \mathrm{~Gb}$ clean data were recovered from the high-throughput sequencing. Human reads (accounting for 99.9\%) were filtered out. The remaining reads were mapped on the genome of $R$. sibirica 246 (GenBank accession no. AABW0100000). Rickettsial unique reads $(\mathrm{n}=266)$ were analyzed against Refseq (https://www.ncbi. nlm.nih.gov/refseq; taxid 766). Most (226/266 [85\%]) 
Table. Homology of 266 identified rickettsial unique reads shared by Rickettsia species, China*

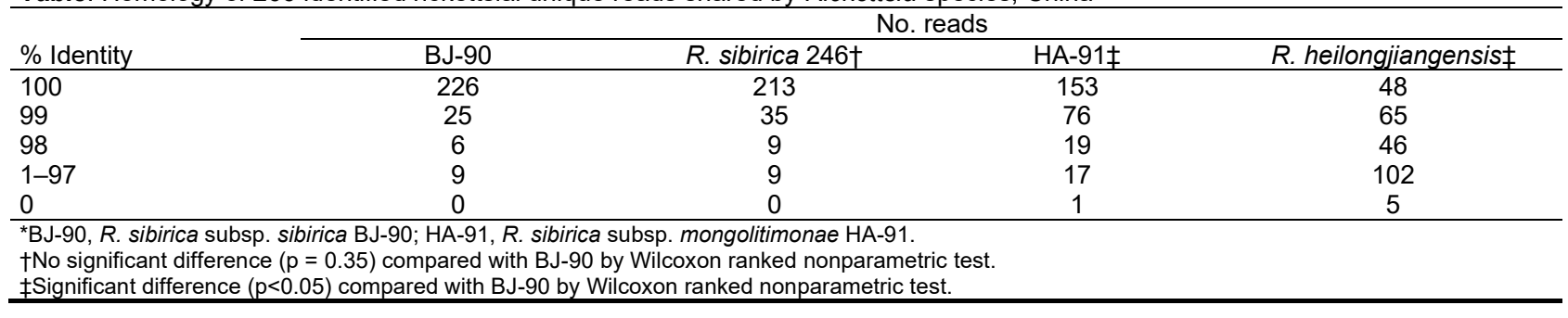

reads were $100 \%$ identical to $R$. sibirica subsp. sibirica BJ-90, whereas 213/266 (80\%) were identical to R. sibirica 246, indicating that the Rickettsia was closest to R. sibirica subsp. sibirica BJ-90 (Table; Appendix Figure 2). Partial sequences of outer membrane protein A, outer membrane protein B, $17 \mathrm{kDa}$ lipoprotein, surface cell antigen 1, and surface cell antigen 4 were amplified with specific primers. Phylogenetic trees showed that the Qinghai sequences clustered with $R$. sibirica subsp. sibirica BJ-90 (Figure; Appendix Figure $3)$. On the basis of next-generation sequencing data and PCR results, we concluded that the causative agent of the patient's infection is closely related to $R$. sibirica subsp. sibirica BJ-90.

We evaluated serum samples from the patient and persons from his surrounding community. Antibodies against $R$. rickettsii (SFG) and $R$. typhi (TG) were determined by indirect immunofluorescence assay. IgG titers of the patient's paired serum samples on day 13 (1:128) and day $167(1: 4,096))$ against SFG were increased by $\geq 4$-fold, suggesting a recent infection with SFG. Approximately $17.4 \%(4 / 23)$ of the serum

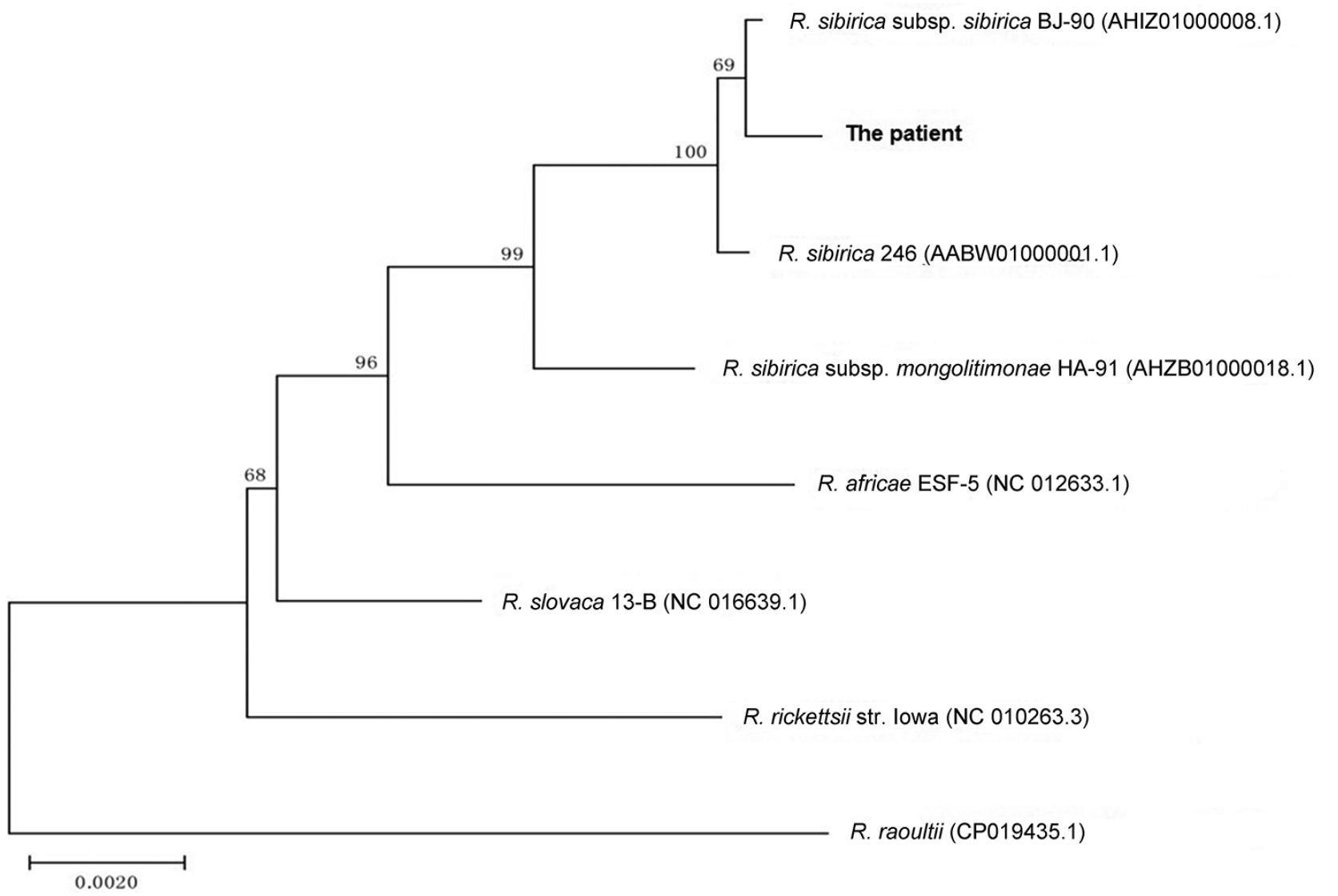

Figure. Phylogenetic analysis of concatenated nucleotide sequences from Rickettsia species collected in 2018 from eschar DNA from a patient in Qinghai Tibet Plateau, China (boldface), and reference sequences. A phylogenetic tree was constructed on the basis of the concatenated partial gltA, ompA, ompB, $17 \mathrm{kDa}$, sca1, and sca4 nucleotide sequences by using the neighbor-joining method with 1,000 bootstrap replicates. Numbers $>70$ indicate the bootstrapping value. GenBank accession numbers listed in Appendix Table 3 (https://wwwnc.cdc.gov/EID/article/27/5/20-3265-App1.pdf). Scale bar represents nucleotide substitutions. 
samples from the local community were positive for SFG, and 4.3\% (1/23) were positive for TG (Appendix Table 1), indicating a high seroprevalence of SFG and co-circulation of TG in the region.

Because of the treating physicians' unawareness of the prevalence of rickettsioses, the patient's illness was misdiagnosed and incorrectly treated. In light of the fatal cases of $R$. sibirica subsp. sibirica infection recently documented in Russia and China (8-10), our report highlights the risk for rickettsial diseases among the public in the Qinghai-Tibet Plateau region and the urgent need for a large-scale seroepidemologic survey.

\section{Acknowledgments}

We thank Pierre Rivailler for analyzing the metagenomic sequences and reviewing this manuscript.

This study was supported by the National Science and Technology Major Projects on Infectious Disease Control and prevention (grant no. 2018ZX10714-002) and Development of Capacity for Pathogen Detection (grant no. 13103110200015003) from the National Institute for Communicable Disease Control and Prevention at the China Centers for Disease Control and Prevention.

\section{About the Author}

Dr. Teng is a research associate at the National Institute for Communicable Disease Control and Prevention, China Centers for Disease Control and Prevention. His research interests include the detection and isolation of rickettsia and the epidemiology of rickettsioses.

\section{References}

1. Merhej V, Angelakis E, Socolovschi C, Raoult D. Genotyping, evolution and epidemiological findings of Rickettsia species. Infect Genet Evol. 2014;25:122-37.

2. Fang LQ, Liu K, Li XL, Liang S, Yang Y, Yao HW, et al. Emerging tick-borne infections in mainland China: an increasing public health threat. Lancet Infect Dis. 2015; 15:1467-79. https:// doi.org/10.1016/S1473-3099(15)00177-2

3. Li J, Hu W, Wu T, Li HB, Hu W, Sun Y, et al. Japanese spotted fever in eastern China, 2013. Emerg Infect Dis. 2018;24:2107-9. https://doi.org/10.3201/ eid2411.170264

4. Abdad MY, Abou Abdallah R, Fournier PE, Stenos J, Vasoo S. A concise review of the epidemiology and diagnostics of rickettsioses: Rickettsia and Orientia spp. J Clin Microbiol. 2018;56:e01728-17. https:/ / doi.org/10.1128/JCM.01728-17

5. Han R, Yang J, Niu Q, Liu Z, Chen Z, Kan W, et al. Molecular prevalence of spotted fever group rickettsiae in ticks from Qinghai Province, northwestern China. Infection, genetics and evolution: journal of molecular epidemiology and evolutionary genetics in infectious diseases. Infect Genet Evol. 2018;57:1-7.

6. Ying L, Zeng-kui L, Gang C, Ming K, Dao-xin L, Yan-ming Z. Identification and phylogenetic analysis of spotted fever group Rickettsia isolated from Qinghai province [in Chinese]. Chin J Vet Sci. 2014;34:1956-61.

7. Jian Y, Li J, Adjou Moumouni PF, Zhang X, Tumwebaze MA, Wang G, et al. Human spotted fever group Rickettsia infecting Yaks (Bos grunniens) in the Qinghai-Tibetan Plateau area. Pathogens. 2020;9:E249. https://doi.org/10.3390/ pathogens 9040249

8. Li H, Fu XY, Jiang JF, Liu RX, Li R, Zheng YC, et al. Severe illness caused by Rickettsia sibirica subspecies sibirica BJ-90 infection, China. Emerg Microbes Infect. 2017;6:e107. https://doi.org/10.1038/emi.2017.95

9. Rudakov N, Samoylenko I, Shtrek S, Igolkina Y, Rar V, Zhirakovskaia E, et al. A fatal case of tick-borne rickettsiosis caused by mixed Rickettsia sibirica subsp. sibirica and "Candidatus Rickettsia tarasevichiae" infection in Russia. Ticks Tick Borne Dis. 2019;10:101278. https:/ / doi.org/ 10.1016/j.ttbdis.2019.101278

10. Jia N, Jiang JF, Huo QB, Jiang BG, Cao WC. Rickettsia sibirica subspecies sibirica BJ-90 as a cause of human disease. N Engl J Med. 2013;369:1176-8. https://doi.org/10.1056/ NEJMc1303625

Address for correspondence: Aiping Qin, State Key Laboratory of Infectious Diseases Prevention and Control, National Institute for Communicable Disease Control and Prevention, Chinese Center for Disease Control and Prevention. 155 Changbai Rd, Changping, Beijing 102206, China; email: qinaiping@icdc.cn

\section{Eosinophilic Meningitis and Intraocular Infection Caused by Dirofilaria sp. Genotype Hongkong}

\author{
Aruna S. Jyotsna, Kollencheri Puthenveettil Vinayan, \\ Lalitha Biswas, Sujithra Haridas, Arun G. Roy, \\ Parasmal Suresh, Anil Kumar
}

Author affiliation: Amrita Institute of Medical Sciences, Amrita Viswa Vidyapeetham Cochin, lindia

DOI: https://doi.org/10.3201/eid2705.203599

Eosinophilic meningitis caused by human diroflarial infection is rare. We report a case of eosinophilic meningitis and concomitant intraocular dirofilarial infection in India. Sequencing of the mitochondrial genome identified the worm as Dirofilaria sp. genotype Hongkong, a close relative of $D$. repens nematodes. 